\title{
The differential diagnosis of ulcerative colitis versus angiodysplasia of the colon with ischemic colitis
}

\author{
Denisa Elena Popa ${ }^{1)}$, Mihaela Calita ${ }^{1)}$, Mihail Cristian PîRlog ${ }^{2)}$, Cristian Constantin ${ }^{3)}$, \\ CRISTIANA GABRIELA POPP ${ }^{4)}$, DAN IONUT GHEONEA ${ }^{1)}$ \\ 1) Department of Gastroenterology, Research Center of Gastroenterology and Hepatology, \\ University of Medicine and Pharmacy of Craiova, Romania \\ 2) Department of Medical Sociology, University of Medicine and Pharmacy of Craiova, Romania \\ 3) Department of Radiology and Medical Imaging, University of Medicine and Pharmacy of Craiova, \\ Romania \\ ${ }^{4)}$ Department of Pathology, Colentina Clinical Hospital, Bucharest, Romania
}

\begin{abstract}
Ulcerative colitis and Crohn's disease are the inflammatory bowel diseases with a continuously increasing of prevalence. Their exact causes are still not well known and, more than that, they are raising up serious issues of diagnosis. The same difficulties of diagnosis are encountered in the case of the colonic angiodysplasia or ischemic colitis (IC). Colonic angiodysplasia is a common vascular abnormality of the gastrointestinal tract, being diagnosed mostly in the elderly persons, in a similar manner to the IC. For all these diseases comorbidities plays their important role both as causes of the onset and aggravating factors during the evolution. The differential diagnosis between these three conditions needs a complex and multidisciplinary approach, involving at least clinical evaluation, endoscopic and imaging assessments, and histopathological exam.
\end{abstract}

Keywords: gastrointestinal tract, inflammatory bowel diseases, endoscopy, angiography, ischemic colitis.

\section{Introduction}

Ulcerative colitis (UC) and Crohn's disease (CD) are two chronic inflammatory bowel (IBD) diseases of unknown origin, affecting the gastrointestinal (GI) tracts of over 2.5 million people in Europe, with continuously rising prevalence in other populations [1]. We are yet to determine a plausible cause of UC; however, it seems to lay on both genetic and environmental factors. Moreover, UC could have similar outcomes during the differential diagnosis to the ischemic colitis (IC), both on clinical and imaging evaluations. IC represents a GI disease which affects the colon by circulatory insufficiency and necrosis of local tissues.

\section{Ulcerative colitis}

$\mathrm{UC}$ is a chronic inflammation of the colonic and rectal mucosa; its evolution being characterized by consecutively episodes of relapses and remissions. The symptoms of UC are dominated by diarrhea (often bloody diarrhea), abdominal pain or cramps, urgency and tenesmus, fever, and weight loss. For an important number of individuals affected by the UC, the extra-intestinal manifestations, such as erythema nodosum, pyoderma gangrenosum, scleritis, uveitis, sacroiliitis, and ankylosing spondylitis are commonly encountered [2].

Inflammation in UC extends from the rectum proximally in the colonic mucosa, with a continuous and symmetric pattern without skipping any area of the colon. The disease typically spars the small bowel, excepting backwash ileitis form that affect up to $10 \%$ of patients and is common in those with concurrent primary sclerosing cholangitis [3, 4].

The diagnosis of UC is primarily based on endoscopic findings of, represented by erythema, loss of the usual vascular pattern, edema, granularity of the mucosa with friability/spontaneous bleeding, erosions, or superficial ulceration. Among these techniques, ileocolonoscopy is the first-line endoscopic procedure in UC, since it allows both the direct visualization of colonic and ileal mucosa and the assessment of disease extent and distribution $[5,6]$.

In UC, ulcers are always surrounded by inflamed mucosa because of its continuous inflammatory nature [7]. The name "ulcerative proctitis" is reserved for cases in whom it involves just the distal part of the colon and the rectum. When it extends from the descending colon towards the rectum, it is considered as limited or distal colitis, while a disease that involves the entire colon is called pancolitis.

Histological features pathognomonic for UC are the following ones: distortion of crypt architecture, neutrophilic cryptitis, even crypt abscesses and ulcers with mucus depletion, basal plasmacytosis. There could be also find an increased number of eosinophils and lymphocytes in the lamina propria [8].

\section{a Angiodysplasia of the colon}

Angiodysplasia currently is the leading vascular abnormality of the GI tract. Its first reporting took place in 1960, when a group of surgeons could successfully

This is an open-access article distributed under the terms of a Creative Commons Attribution-NonCommercial-ShareAlike 4.0 International Public License, which permits unrestricted use, adaptation, distribution and reproduction in any medium, non-commercially, provided the new creations are licensed under identical terms as the original work and the original work is properly cited. 
identify and link a vascular malformation in the cecum with the massive bleeding in a 69-year-old woman, through operative mesenteric arteriography [9].

It is the second cause of lower GI bleeding in patients older than 60 years after diverticulosis, and the most common reason for occult GI bleeding, representing approximately $6 \%$ of all cases of lower GI bleeding.

Patients with angiodysplasia usually present with maroon-colored stool, melena, hematochezia or hemoccult positive stool. Bleeding is usually low grade, but it can be massive in approximately $15 \%$ of patients and although stops spontaneously in greater than $90 \%$ of cases, is often recurrent $[10,11]$.

The exact pathophysiological mechanism of the development of this vascular abnormality is unknown, but chronic venous obstruction may play a role. The theory of chronic venous obstruction suggests that gradual dilation of the submucosal veins, dilation of the venules and arteriolar capillary units feeding them leads to capillary rings dilation, the precapillary sphincters lose their competency, and a small arteriovenous communication form.

All these lead to the characteristic early-filling veins that were noticed during mesenteric angiography [12], while other studies have also described the portal hypertension colopathy, a form of colonic angiodysplasia [13].

There are also other theories about the etiology of angiodysplasia, that include degenerative changes of the small blood vessels that seem to be associated with increasing age and long-term local hypo-oxygenation of the microcirculation due to cardiac, vascular, pulmonary diseases or liver cirrhosis [14].

Epidemiological studies have shown an estimated prevalence rate of $0.8 \%$ for angiodysplasia, in healthy patients, older than 50 years, who are undergoing screening colonoscopy. Moreover, multiple angiodysplastic lesions in the colon are more frequently than single ones [15].

The most useful diagnostic technique for angiodysplasias is the selective mesenteric angiography, especially in patients with massive bleeding, when the common colonoscopy became difficult for establishing a diagnosis. Thus, through the mesenteric angiography, the earliest signs that are observed are opacified, dilated, and slowly emptying draining vein within the intestinal wall. The latest sign of angiodysplasia is observed in $60-70 \%$ of the cases, and is represented by an early-filling vein, that may be found in the arterial phase, indicating a more developed arteriovenous communication through the angiodysplastic lesion [16]. As we mentioned above, the endoscopic appearance of angiodysplasia may be difficult to discern from spider angioma, radiation injury, UC, CD, IC, portal hypertensive colopathy and suction artifacts.

Histologically features of angiodysplasia are dilated, distorted, thin-walled vessels, while the markedly dilated submucosal vasculature is the most consistent abnormality and one of the earliest changes identified.

\section{ㅁ Ischemic colitis}

IC was initially described in 1963 [17] and term was first used in 1966 [18]. The disease was defined as a clinical entity characterized by circulatory insufficiency of the colon, local tissue necrosis and systemic manifestations [19]. IC is more commonly found in elderly patients, usually those with significant comorbidities from the spectrum of cardiovascular conditions, diabetes mellitus, chronic renal failure or coagulopathies [20]. Long-distance running, illicit drug use (especially cocaine abuse), collagen vascular disease, hematological disorders and medication-induced colonic ischemia have been also reported as presumable causes of IC, both in young and old patients [21]. These clinically risk factors are important to be considered when a patient's condition is evaluated for the diagnosis of IC, and to them could be added also demographic risk factor such as age and female gender, that seems to increase the risk of developing IC [22]. In the category of young patients diagnosed with IC the most predominant are female which often present a relatively acute symptomatology. An opposite situation could be found in elderly patients, where the indolent presentation is more commonly.

For IC were identified two distinct forms. The first one is the occlusive IC, while the second is a nonocclusive form of the diseases. Both clinical forms may involve the territory of the inferior mesenteric artery, colonic branches of the superior mesenteric artery and superior and inferior mesenteric veins. Although, IC is generally viewed as a non-occlusive form of intestinal ischemia.

Despite the large number of studies, the epidemiological data related to the incidence and prevalence of IC in the general population are still unknown. The most invoked cause for this loss of data is that many cases of transient ischemic injury remained undiagnosed, even if they are gastroenterological assessed. The aspect that it is most interesting for us is that other studies underlined that many cases of IC are misdiagnosed as IBD, and they are considered and treated like that [23].

It was showed that IC is more common in the left colon. The most important reason that stand behind this observation is that the splenic flexure of the colon is therefore a watershed area (Griffiths' point), and it is particularly susceptible to ischemic injury. This kind of injuries are met in the approximately $5 \%$ of individuals in whom the marginal artery of Drummond is thinned or absent and also in the rectosigmoid junction (Sudeck's point) [24]. Right colon IC is a rare phenomenon, and is mainly due to low-flow states, and repeated hypotensive episodes that led to vasoconstriction of the vasa recta in the right colon.

The IC is also common in the patients who have chronic renal failure and are on hemodialysis, these clinical complications being associated with poor prognosis of this colon disease [25].

A very rare entity of the same kind as IC is the ischemic proctosigmoiditis, that it seems to be caused by the abundant collateral blood supply located within the pelvis and perineum and when this entity occurs, a precipitating factor is often identified [26].

Another type of IC classification is based on clinical findings, and accordingly, there are two other forms of IC: the nongangrenous type, subdivided in turn into transient reversible colitis and a chronic form, and the gangrenous 
type. For the first category, the nongangrenous form, the histological findings have shown some self-limiting damage of the mucosa and submucosa, that manifests as edema, submucosal hemorrhage and/or partial mucosal necrosis with ulceration. The individuals affected by this form may also have clinically lower GI bleeding.

The clinical manifestation of IC varies with the severity degree of the condition. There were described three degrees the progressive severity (mild, intermediate, respectively severe), but it was also found that more than $50 \%$ of all patients with IC experience the mild form of the disease. In the mild form of the IC, only the colonic mucosa and submucosa are affected and is clinically characterized by abdominal pain, distension, bloody diarrhea, and resolution of symptoms with conservative nonoperative measures without long-term sequelae [27]. In the intermediate form of IC, it was noticed a variable thickness of the muscularis, and those patients may experience stricture subsequently or persistent colitis and require resection. The severe form of the IC affects $10 \%$ to $20 \%$ of all patients and it is characterized by transmural gangrenous necrosis of the colonic segment, that rapidly leads to peritonitis urging surgical resection. Colonic distention represents the clinical marker for the severe involvement [28, 29].

The differential diagnosis of IC is a difficult one in some cases because the imaging evaluation plays a mostly adjunctive role. Laboratory tests for IC diagnosis are all nonspecific and relatively late findings and they assess marked leukocytosis, serum acidosis and increased lactate or lactate dehydrogenase (LDH) levels.

The endoscopic examination represents the most frequently and useful tool for definitive diagnosis of IC. During the acute phase, through the endoscopy could be notice a pale mucosa with small areas of petechial hemorrhage which is eloquent for a mild ischemia. The more severe disease is endoscopically revealed by a progressively darker mucosa (dark blue to black) and/or ulceration [30].

One of the frequent issues that are meeting during the diagnosis process of IC by endoscopic examination, it is represented by the difficulty to distinguish between the IC forms and other colitis, such as CD, UC, infectious colitis, or radiation colitis. More than that, in elderly patients, the differential diagnosis between IC and UC may be even more difficult, but one of the useful observations is the rectal preservation which makes the difference to the UC. To these diagnosis techniques it is added the biopsy for histological examination, which could make the difference between these above-mentioned clinical entities. From the histologically point of view, IC is characterized by crypt abnormalities, sloughing of epithelial cells, edema, capillary thrombosis, and the presence of quite a few inflammatory cells [31].

Imaging evaluations have their important role in the diagnosis, but it has been proven that computed tomography (CT) scans may be fully normal in patients with early IC. The most common CT finding is nonspecific and consists of moderate circumferential wall thickening caused by the edema or hemorrhage. Certain findings like pneumatosis are not pathognomonic but raise the possibility of IC
[32]. Finally, the angiography plays an important role in the diagnosis of IC, but also have a specific therapeutically tool for this disease.

Not only the very technically approaches of the diagnosis are important in the case of IC, but also the high index of suspicion for the disease, based on the clinical sense of the gastroenterologist, the presence of comorbidities, and the history of GI manifestation. Correct interpretation of the symptoms, signs, and laboratory test results associated with colonic ischemia must be followed by prompt imaging, endoscopy, and/or operative exploration, depending on the severity of disease.

\section{ㅁ Differential diagnosis process}

The difficulties of the differential diagnosis between $\mathrm{UC}$, angiodysplasia of the colon and IC are very common and provocative for the gastroenterologists and could be significantly illustrated by the following clinical case.

The 65-year-old male patient was known since 2007 with type II diabetes mellitus, ethanolic liver cirrhosis and esophageal varices since 2010, and chronic hypertension ( $3^{\text {rd }}$ grade). During the last 12 months, he was several times admitted to the Clinic of Gastroenterology, Emergency County Hospital, Craiova, Romania, accusing pain in the lower abdominal floor, acceleration of the intestinal transit, rectal bleeding, weight loss, physical asthenia, and fatigue. These symptoms firstly led the clinicians to the suspicion of infectious colitis diagnosis. To confirm this suspicion, were done ova and parasite exams of the stool, which had negative results and thus infirmed the suspicion of infectious colitis.

Also, it was done the abdomino-pelvic ultrasound which does not reveal significant changes for the presumable intestinal diagnosis: liver with irregular contour, inhomogeneous, hyperechogenic, with no localized processes.

Due to the presence of rectal bleeding, weight loss and patient's old age it was decided to carry out an inferior digestive endoscopy that revealed the following changes: ileocecal valve with edema and hyperemia, vascular dilatations, splenic flexure with edema and erythema, and at the level of the rectosigmoid junction several areas of necrosis and several linear ulcers (Figure 1). Concomitant with the endoscopy were collected biological samples for the histopathological (HP) examination from the patient's affected areas.

The reveals of endoscopy imposed new investigations to establish a certain diagnosis and based on that to start as early as possible the treatment. Thus, it was realized a CT Siemens 20 slice exam with automatic injection bolus. The results of this examination were the following ones:

- thorax: no pathological changes, except for an infracentimetric thyroid node;

- abdomen: liver with irregular contour and regeneration nodules;

- pelvis (rectosigmoid area): a marked parietal thickening, iodophilic with infiltration of adjacent peritoneal fat (Figure 2, A and B) with tortuous vascular paths and locoregional inflammatory adenopathy with a maximum size of $1.2 / 0.9 \mathrm{~cm}$ (Figure $2 \mathrm{C}$ ). 
The results of the CT exam have not been proven to be pathognomonic for a certain diagnosis; thus, it was decided to be carried out a supplementary evaluation, the angiography.

The angiography of the superior mesenteric artery and inferior mesenteric artery had revealed for both areas an early crossing of the contrast substance from the arterial system to the venous system (Figure 3 ). The same procedure led to the suspicion of angiodysplasia in the small and respectively large intestine sections that were angiographically examined.

Finally, the results of the HP examination have shown a colonic mucosa with an ulceration area, granulation, and reepithelialization tissue; chorion with minimal inflammatory infiltrate and moderate/marked fibrosis (Figure 4, A-C). From the HP point of view, these results are highly suggestive for the diagnosis of IC, possibly a consequence of the type II diabetes mellitus.

\section{Conclusions}

The diseases of the GI tract that are including rectal bleeding as main symptom still represents a challenge for clinicians and an important health issue for the individuals affected. The presence of this clinical manifestation requires a rapid and clear diagnosis process which involves a multidisciplinary approach. The differential diagnosis between $\mathrm{UC}$, colonic angiodysplasia and IC, needs to be done in subsequently stages involving clinical evaluation, endoscopic and imaging assessments, and HP exam. Another important aspect that needs to be considered is the evaluation of the comorbidities and their influence on the GI disease.

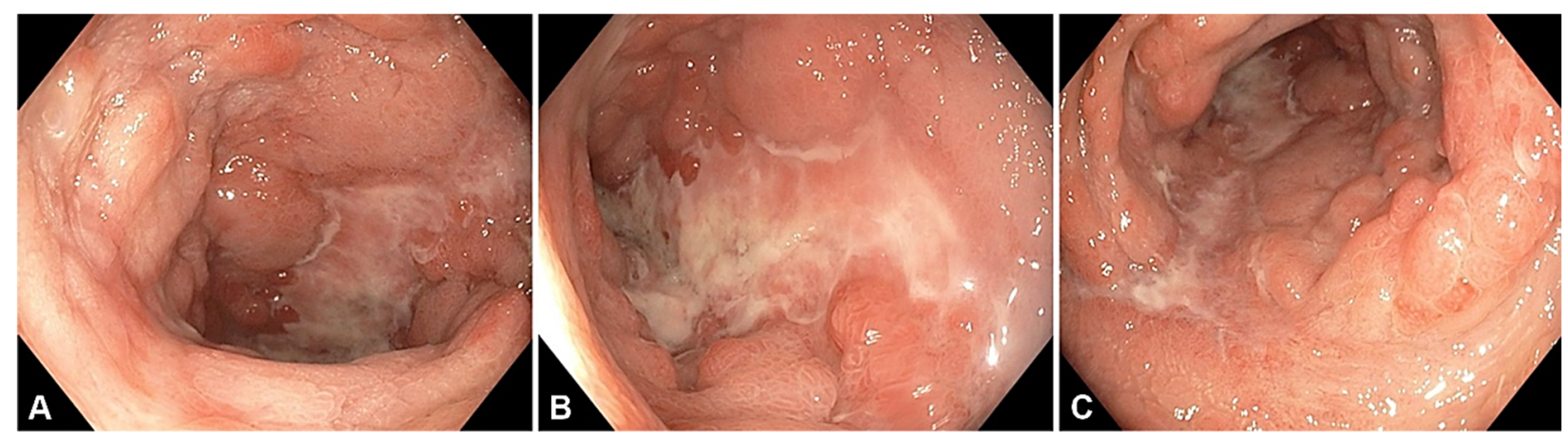

Figure $1-(A-C)$ Endoscopy images showing the rectosigmoid junction with linear ulcers and mucosal edema and erythema.
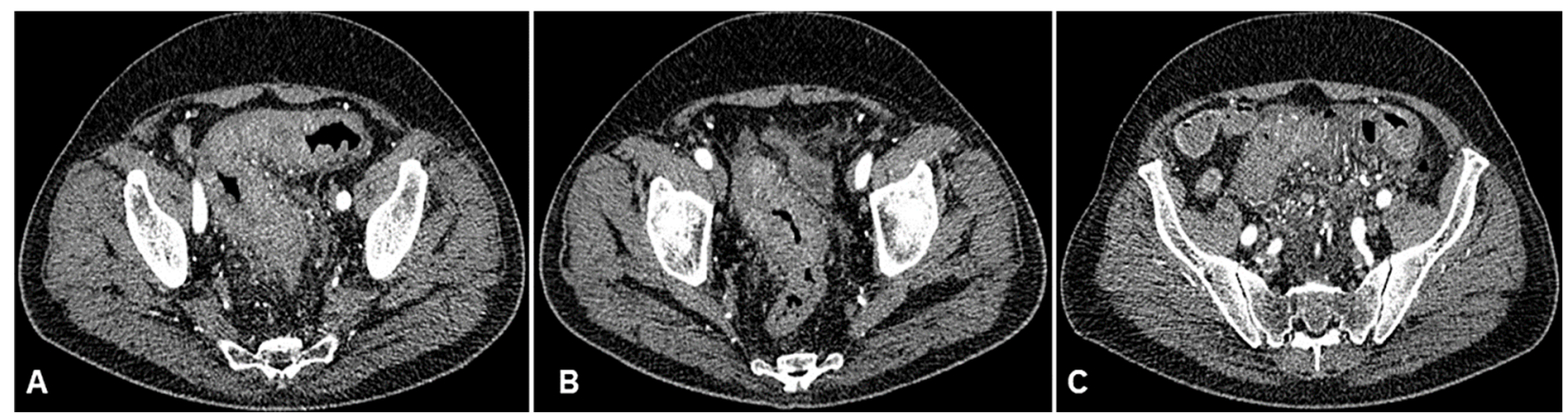

Figure 2-(A and B) Marked parietal thickening, iodophilic with infiltration of adjacent peritoneal fat, tortuous vascular paths; (C) Locoregional inflammatory adenopathy (CT Siemens 20 slice). CT: Computed tomography.
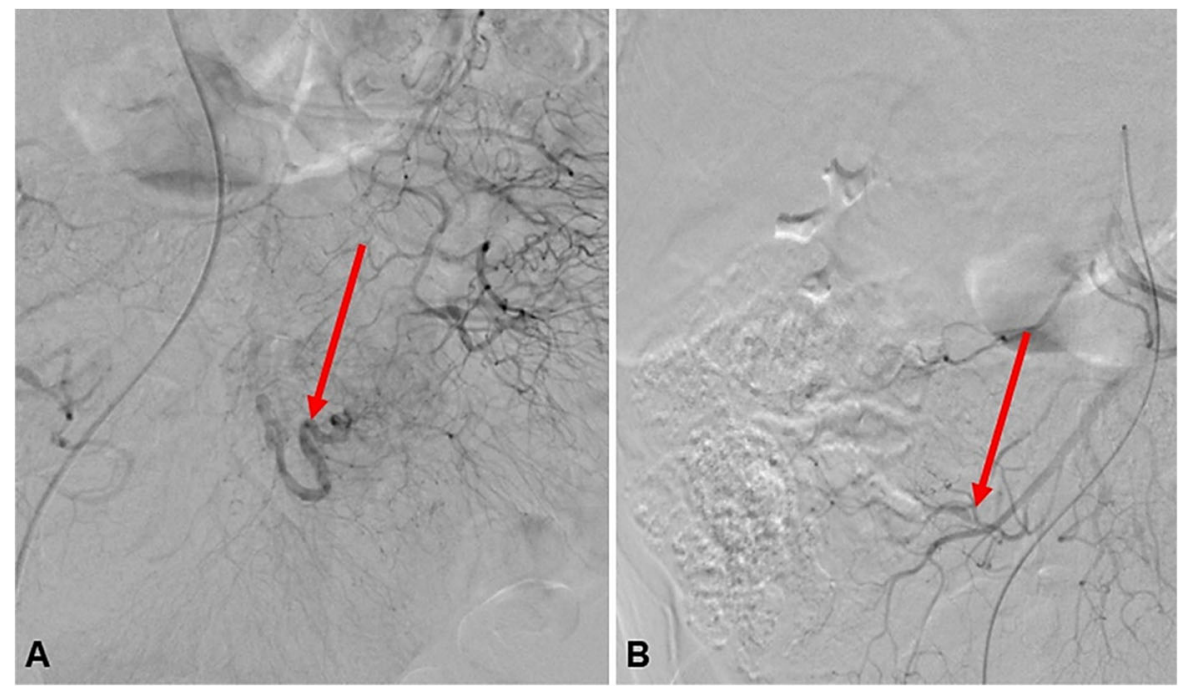

Figure 3 - Angiography images of the superior mesenteric artery $(A)$, respectively inferior mesenteric artery $(B)$ showing an early crossing of the contrast substance from the arterial system to the venous system. 


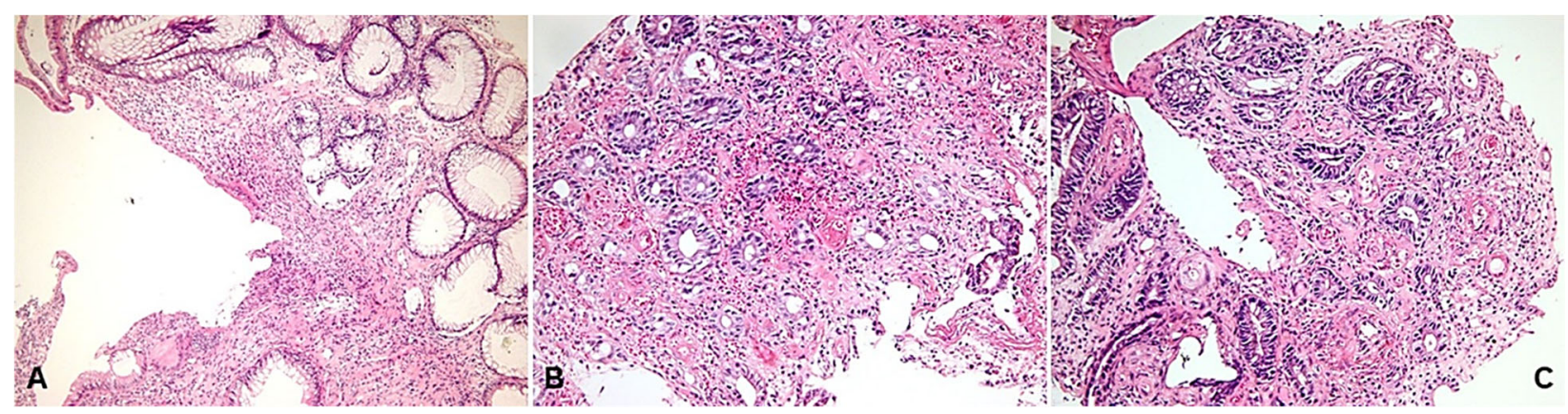

Figure 4-(A) Colonic mucosa with an ulceration area, granulation, and re-epithelialization tissue; chorion with minimal inflammatory infiltrate and moderate/marked fibrosis; (B) Colonic mucosa with regenerative epithelial changes; chorion with frequent fibroblasts/fibrocytes, areas of hemorrhage and moderate/marked fibrosis; numerous capillaries with intramural fibrinoid necrosis and/or intraluminal fibrinous thrombi; (C) Colonic mucosa with regenerative epithelial changes; chorion with frequent fibroblasts/fibrocytes, areas of hemorrhage and moderate/marked fibrosis; numerous capillaries with intramural fibrinoid necrosis and endothelial proliferation. Hematoxylin-Eosin $(H E)$ staining: $(A) \times 100$; $(B$ and $C) \times 200$.

\section{Conflict of interests}

The authors declare that they have no conflict of interests.

\section{Authors' contribution}

Denisa Elena Popa \& Mihaela Caliţa equally contributed to this article and share main authorship.

\section{References}

[1] Molodecky NA, Soon IS, Rabi DM, Ghali WA, Ferris M, Chernoff G, Benchimol El, Panaccione R, Ghosh S, Barkema HW, Kaplan GG. Increasing incidence and prevalence of the inflammatory bowel diseases with time, based on systematic review. Gastroenterology, 2012, 142(1):46-54e42; quiz e30. https://doi.org/10.1053/j.gastro.2011.10.001 PMID: 22001864

[2] Levine JS, Burakoff R. Extraintestinal manifestations of inflammatory bowel disease. Gastroenterol Hepatol (NY), 2011, 7(4):235-241. PMID: 21857821 PMCID: PMC3127025

[3] Rimoin DL, Pyeritz RE, Korf BR (eds). Emery and Rimoin's principles and practice of medical genetics. $6^{\text {th }}$ edition, Academic Press-Elsevier, Amsterdam, 2013, 1-38. https://www.elsevier. com/books/emery-and-rimoins-principles-and-practice-of-medicalgenetics/rimoin/978-0-12-383834-6

[4] Haskell H, Andrews CW Jr, Reddy SI, Dendrinos K, Farraye FA, Stucchi AF, Becker JM, Odze RD. Pathologic features and clinical significance of "backwash" ileitis in ulcerative colitis. Am J Surg Pathol, 2005, 29(11):1472-1481. https://doi.org/ 10.1097/01.pas.0000176435.19197.88 PMID: 16224214

[5] Bharadwaj S, Narula N, Tandon P, Yaghoobi M. Role of endoscopy in inflammatory bowel disease. Gastroenterol Rep (Oxf), 2018, 6(2):75-82. https://doi.org/10.1093/gastro/goy006 PMID: 29780594 PMCID: PMC5952948

[6] Leighton JA, Shen B, Baron TH, Adler DG, Davila R, Egan JV, Faigel DO, Gan SI, Hirota WK, Lichtenstein D, Qureshi WA, Rajan E, Zuckerman MJ, VanGuilder T, Fanelli RD; Standards of Practice Committee, American Society for Gastrointestinal Endoscopy. ASGE Guideline: endoscopy in the diagnosis and treatment of inflammatory bowel disease. Gastrointest Endosc, 2006, 63(4):558-565. https://doi.org/10.1016/j.gie. 2006.02.005 PMID: 16564852

[7] Rameshshanker R, Arebi N. Endoscopy in inflammatory bowel disease when and why. World J Gastrointest Endosc, 2012, 4(6):201-211. https://doi.org/10.4253/wjge.v4.66.201 PMID: 22720120 PMCID: PMC3377861

[8] Cerilli LA, Greenson JK. The differential diagnosis of colitis in endoscopic biopsy specimens: a review article. Arch Pathol Lab Med, 2012, 136(8):854-864. https://doi.org/10.5858/arpa. 2012-0205-RA PMID: 22849731

[9] Margulis AR, Heinbecker P, Bernard HR. Operative mesenteric arteriography in the search for the site of bleeding in unexplained gastrointestinal hemorrhage: a preliminary report. Surgery, 1960, 48(3):534-539. PMID: 14421054
[10] Oancea CN, Butaru AE, Streba CT, Pirici D, Rogoveanu I, Diculescu MM, Gheonea DI. Global hepatitis C elimination: history, evolution, revolutionary changes and barriers to overcome. Rom J Morphol Embryol, 2020, 61(3):643-653. https://doi.org/10.47162/RJME.61.3.02 PMID: 33817705 PMCID: PMC8112794

[11] Popa P, Streba CT, Caliţă M, lovănescu VF, Florescu DN, Ungureanu BS, Stănculescu $A D$, Ciurea RN, Oancea $C N$, Georgescu D, Gheonea DI. Value of endoscopy with narrowband imaging and probe-based confocal laser endomicroscopy in the diagnosis of preneoplastic lesions of gastrointestinal tract. Rom J Morphol Embryol, 2020, 61(3):759-767. https://doi.org/ 10.47162/RJME.61.3.14 PMID: 33817717 PMCID: PMC8112779

[12] Junquera F, Saperas E, de Torres I, Vidal MT, Malagelada JR. Increased expression of angiogenic factors in human colonic angiodysplasia. Am J Gastroenterol, 1999, 94(4):1070-1076. https://doi.org/10.1111/j.1572-0241.1999.01017.x PMID: 10201485

[13] Biss T, Hamilton P. Myelofibrosis and angiodysplasia of the colon: another manifestation of portal hypertension and massive splenomegaly? J Clin Pathol, 2004, 57(9):999-1000. https:// doi.org/10.1136/jcp.2003.015479 PMID: 15333669 PMCID: PMC1770404

[14] Heyde EC. Gastrointestinal bleeding in aortic stenosis. N Engl J Med, 1958, 259:196. https://doi.org/10.1056/NEJM195807 242590416 https://www.nejm.org/doi/full/10.1056/NEJM1958 07242590416

[15] Foutch PG, Rex DK, Lieberman DA. Prevalence and natural history of colonic angiodysplasia among healthy asymptomatic people. Am J Gastroenterol, 1995, 90(4):564-567. PMID: 7717311

[16] Emanuel RB, Weiser MM, Shenoy SS, Satchidanand SK, Asirwatham J. Arteriovenous malformations as a cause of gastrointestinal bleeding: the importance of triple-vessel angiographic studies in diagnosis and prevention of rebleeding. J Clin Gastroenterol, 1985, 7(3):237-246. https://doi.org/10. 1097/00004836-198506000-00010 PMID: 3874898

[17] Boley SJ, Schwartz S, Lash J, Sternhill V. Reversible vascular occlusion of the colon. Surg Gynecol Obstet, 1963, 116:5360. PMID: 13968597

[18] Marston A, Pheils MT, Thomas ML, Morson BC. Ischaemic colitis. Gut, 1966, 7(1):1-15. https://doi.org/10.1136/gut.7.1.1 PMID: 5906128 PMCID: PMC1552376

[19] Toursarkissian B, Thompson RW. Ischemic colitis. Surg Clin North Am, 1997, 77(2):461-470. https://doi.org/10.1016/s00 39-6109(05)70561-2 PMID: 9146725

[20] Brandt LJ, Boley SJ, Mitsudo S. Clinical characteristics and natural history of colitis in the elderly. Am J Gastroenterol, 1982, 77(6):382-386. PMID: 7091124

[21] Marston A. Acute intestinal ischaemia. BMJ, 1990, 301(6762): 1174-1176. https://doi.org/10.1136/bmj.301.6762.1174 PMID: 2261552 PMCID: PMC1664320

[22] Higgins PDR, Davis KJ, Laine L. Systematic review: the epidemiology of ischaemic colitis. Aliment Pharmacol Ther, 
2004, 19(7):729-738. https://doi.org/10.1111/j.1365-2036.2004. 01903.x PMID: 15043513

[23] Brandt LJ, Boley SJ. Colonic ischemia. Surg Clin North Am, 1992, 72(1):203-229. https://doi.org/10.1016/s0039-6109(16) 45635-5 PMID: 1731384

[24] Scharff JR, Longo WE, Vartanian SM, Jacobs DL, Bahadursingh AN, Kaminski DL. Ischemic colitis: spectrum of disease and outcome. Surgery, 2003, 134(4):624-629; discussion 629630. https://doi.org/10.1016/s0039-6060(03)00308-8 PMID: 14605623

[25] Cappell MS. Intestinal (mesenteric) vasculopathy. II. Ischemic colitis and chronic mesenteric ischemia. Gastroenterol Clin North Am, 1998, 27(4):827-860, vi. https://doi.org/10.1016/ s0889-8553(05)70034-0 PMID: 9890115

[26] Bharucha AE, Tremaine WJ, Johnson CD, Batts KP. Ischemic proctosigmoiditis. Am J Gastroenterol, 1996, 91(11):23052309. PMID: 8931407
[27] Boley SJ, Brandt LJ, Veith FJ. Ischemic disorders of the intestines. Curr Probl Surg, 1978, 15(4):1-85. https://doi.org/ 10.1016/s0011-3840(78)80018-5 PMID: 365467

[28] Boley SJ. 1989 David H. Sun lecture. Colonic ischemia - 25 years later. Am J Gastroenterol, 1990, 85(8):931-934. PMID: 2375319

[29] Jonas J, Böttger T. Diagnostik und Prognose des Mesenterialinfarktes [Diagnosis and prognosis of mesenterial infarct]. Med Klin (Munich), 1994, 89(2):68-72. PMID: 8164598

[30] Andrus $\mathrm{CH}$. Endoscopic assessment of vascular disorders. Semin Colon Rectal Surg, 1994, 5:27-31.

[31] Barnett MG, Longo WE. Intestinal ischemia after aortic surgery. Semin Colon Rectal Surg, 1993, 4:229-234.

[32] Jacobs JE, Birnbaum BA. CT of inflammatory disease of the colon. Semin Ultrasound CT MR, 1995, 16(2):91-101. https:// doi.org/10.1016/0887-2171(95)90002-0 PMID: 7794608

\section{Corresponding author}

Mihail Cristian Pîrlog, Associate Professor, PhD, Department of Medical Sociology, University of Medicine and Pharmacy of Craiova, 2 Petru Rareş Street, 200349 Craiova, Dolj County, Romania; Phone +40722-642 561, e-mail: mihail.pirlog@umfcv.ro

Received: August 11, 2021

Accepted: December 29, 2021 